\title{
IDENTIFICATION OF TRACEABILITY BARCODE BASED ON PHASE CORRELATION ALGORITHM
}

Liying Lang ${ }^{1, *}$, Xiaofang Zhang ${ }^{2}$

${ }^{1}$ Hebei University of Engineering, Handan, China, 056038;

${ }^{2}$ College of Information and Electrical Engineering, Hebei University of Engineering, Handan, 056038, China

* Corresponding author, Address: Hebei University of Engineering, Handan,Hebei province, Guangming South Street 199, 056038 , P. R. China, Tel: 0310-8579551, Fax:03108578746, Email: langliying@126.com

Abstract: In the paper phase correlation algorithm based on Fourier transform is applied to the traceability barcode identification, which is a widely used method of image registration. And there is the rotation-invariant phase correlation algorithm which combines polar coordinate transform with phase correlation, that they can recognize the barcode with partly destroyed and rotated. The paper provides the analysis and simulation for the algorithm using Matlab, the results show that the algorithm has the advantages of good real-time and high performance. And it improves the matching precision and reduces the calculation by optimizing the rotation-invariant phase correlation.

Keywords: traceability barcode recognition, Fourier transform, phase correlation, image registration

\section{INTRODUCTION}

At present in our country, the major food output such as cereals, fruits, meat, eggs and aquatic products occupy first in the world. In order to guarantee the people's food security and effectively control of food-borne disease outbreak, the work to establish food tracking and retrospect will have a tremendous impact on the development of the food industry in China.

Please use the following format when citing this chapter:

Lang, L. and Zhang, X., 2009, in IFIP International Federation for Information Processing, Volume 295, Computer and Computing Technologies in Agriculture II, Volume 3, eds. D. Li, Z. Chunjiang, (Boston: Springer), pp. 1721-1727. 
Compared the way of $\log$ file retrospect (Tillett R D, 1991), it is more efficient, real-time and convenient to make use of the barcode superiority characteristic to manage the agricultural product security and the retrospect. This way has many merits (Paulsen, 1997). It not only provides the completely transparent managed capacity in the agricultural product supply chain, ensures safety of agricultural products throughout visual control, monitoring and retrospect, but also may monitor the planter and cultivation source pollution, the chemical additive and deleterious substance in the process of production produce, the security risks in the circulation link. However, there will inevitably appear the attrition and the distortion of the traceability barcode in the course of agricultural product circulation. For this purpose, the phase correlation algorithm which has powerful anti-geometric deformation capacity to the barcode identification is applied to deal with this problem.

\section{BASAL PRINCIPLE OF ALGORITHM}

Phase correlation algorithm is non-linear in frequency domain, which is based on Fourier transform (Stone, 2001). It makes use of a number of important properties of the image in frequency domain, such as translation, rotation, zooming invariance characters (Srinivasa R B, 1996), that is to say there exists corresponding relation between the airspace and the frequency domain when there is translation, rotation, zooming between the images. Fast Fourier Transform a large extent improves the operation efficiency of the algorithm, and it behaves a good robust to the noise of the frequency correlation. Therefore phase correlation algorithm is a more effective method to achieve the image registration.

\subsection{The theory estimation of translating parameter}

Hypothesize $f_{1}(x, y)$ and $f_{2}(x, y)$ are two one-dimensional barcodes which have definitely translating relation (Kobayashi, 1996). They satisfy with formula (1). (That is to say, $f_{2}(x, y)$ results from simply translating by $\left.f_{1}(x, y)\right)$

$$
f_{2}(x, y)=f_{1}\left(x-x_{0}, y-y_{0}\right)
$$

The character according to Fourier transform can may:

$$
F_{2}(u, v)=F_{1}(u, v) e^{-j\left(u x_{0}+v y_{0}\right)}
$$


In above formula, $F_{1}(u, v)$ and $F_{2}(u, v)$ are neutralized respectively Fourier transform drawing from $f_{1}(x, y)$ and $f_{2}(x, y)$.Their cross-power spectrum is that:

$$
\frac{F_{1}^{*}(u, v) F_{2}(u, v)}{\left|F_{1}^{*}(u, v) F_{2}(u, v)\right|}=e^{-j\left(u x_{0}+v y_{0}\right)}
$$

$F_{1}^{*}(u, v)$ is complex conjugation of $F_{1}(u, v)$ in style. The formula (4) carries out Fourier transform on the contrary alternation but gets a twodimensional pulse function. That is:

$$
F^{-1}\left(e^{-j\left(u x_{0}+v y_{0}\right)}\right)=\delta\left(x-x_{0}, y-y_{0}\right)
$$

It can find that, phase correlation function at the surface all is zero apart from $\delta$ function, which is the location of their displacement value $\left(x_{0}, y_{0}\right)$ (Harold S S,2002). The function peak value should be 1 under ideal circumstances, but due to noise and other reasons, its peaks often less than 1 . The peak value depends on whether two images mate the degree goodness or badness.

\subsection{The theory estimation of rotating parameter}

Hypothesize $f_{1}(x, y)$ and $f_{2}(x, y)$ are two one-dimensional barcode images. $f_{2}(x, y)$ results from simply rotating by $f_{1}(x, y)$. That is to say:

$$
f_{2}(x, y)=f_{1}\left(x \cos \theta_{0}+y \sin \theta_{0},-x \sin \theta_{0}+y \cos \theta_{0}\right)
$$

According to the rotation of Fourier transform, the relationship between the two images after shift as follows:

$$
F_{2}(u, v)=F_{1}\left(u \cos \theta_{0}+v \sin \theta_{0},-u \sin \theta_{0}+v \cos \theta_{0}\right)
$$

From above formula it can see that the image of the space domain in the rotation $\theta_{0}$ perspective, the corresponding Fourier transform in the frequency domain is the same rotation angle $\theta_{0}$. Due to Cartesian coordinates corresponding to the rotation angle of the polar coordinate translation (Wolberg, 2000), it will be (7) converted to the form of polar coordinates as follows:

$$
F_{2}(\rho, \theta)=F_{1}\left(\rho, \theta-\theta_{0}\right)
$$


In the formula, $\rho=\sqrt{u^{2}+v^{2}}, \theta=\arctan \frac{v}{u}$, from formula (7) it can find that they satisfy a simple translation relation between $F_{2}(\rho, \theta)$ and $F_{1}(\rho, \theta)$. Make use of above-mentioned phase correlation method to be ok to gain the revolution angle.

\section{EXPERIMENT AND RESULT ANALYSIS}

According to situation which the barcode possibly appears in the actual circulation, we divide the experiments into two following parts: (1) When the traceability barcode is partly frayed or masked, translation parameters are estimated based on phase correlation algorithm; (2) Using the rotationinvariant phase correlation algorithm to estimate the rotation parameters when the barcode revolves slightly.

\subsection{Translation experiment}

In the process of algorithm simulation, the paper first pretreats the images using median filter to filter out pulse interference and image scanning noise, and uses MATLAB tools to simulate the image of one-dimensional barcodes. The results show that the phase correlation algorithm only has good effect on translation image detection, even if only in the relevant content of $1 / 4$, the success rate is still over $90 \%$. There are two barcode images of mutual translation. The two figures with relevant contents of half and quarter were compared. The results clearly show that with the relevant content reducing gradually the peak values reduce sharply, as shown in figure 1 and figure 2 . The relevant peak soon declines when there exists rotating in the images .However, it has a better match rate within only 1.5 degrees, more than 3 degrees the algorithm completely ineffective.

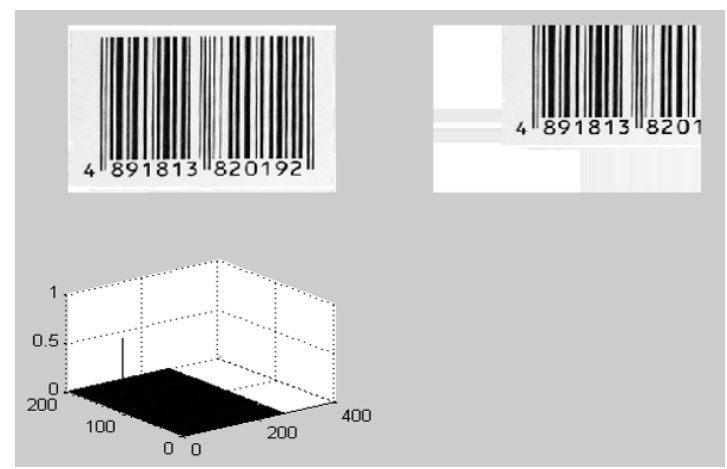

Fig.1: Correlation result of half related 


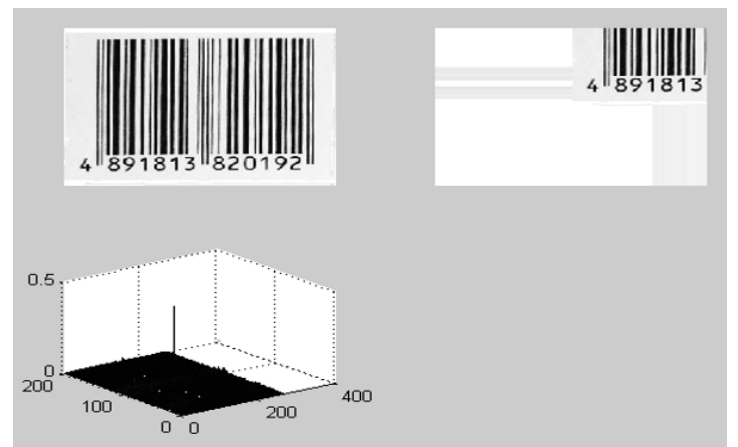

Fig.2: Correlation result of quarter related

\subsection{Rotation experiment}

The algorithm is carried out a certain amount of optimization when in the process of rotation-invariant phase correlation algorithm simulation. In the polar coordinate mapping, it first seeks admission points in a very homogeneous coordinates in turn to obtain the value in the Cartesian coordinate system mapping, so that it may sample around the neighborhood part of origin point in the Cartesian coordinate system. Because after the Fourier transform and centering the image energy mainly concentrates in the neighborhood of the origin, it can reduce the mapping error from Cartesian to polar coordinates. The experiment results prove that it can have great correlation peak growth. So that it will not only improve the matching accuracy but also greatly reduce the computation of following stage. Below are the barcodes with different rotational angle carried out the simulation. Figure 3-a and figure 4-a are polar coordinate transform of the original and rotating barcodes, corresponding correlation result in figure 3-b and figure 4b. The results show that the smaller the angle of deformation registration the results more accurate.

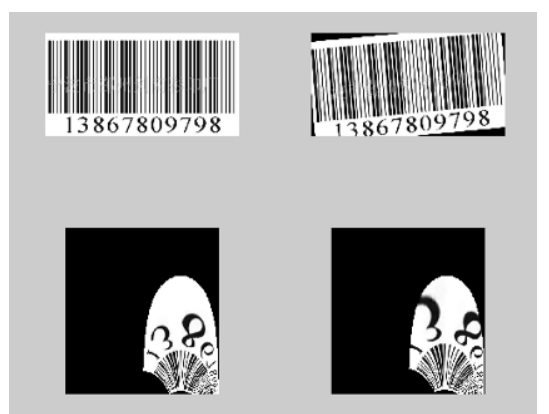

(a) polar coordinate transforms

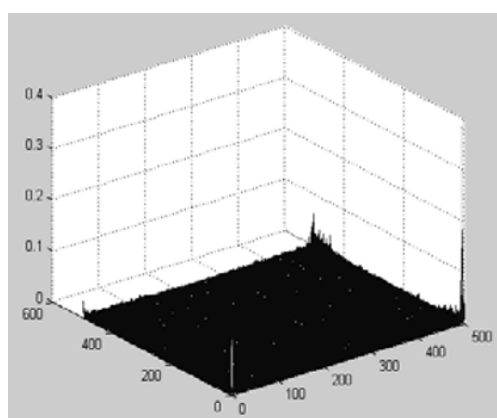

(b) correlation result

Fig.3: Result of rotating angle of 5 


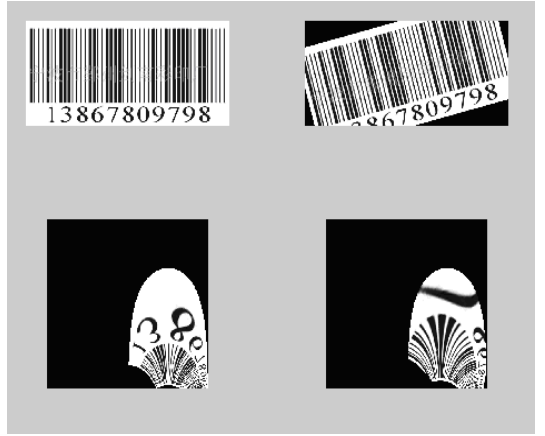

(a) polar coordinate transforms

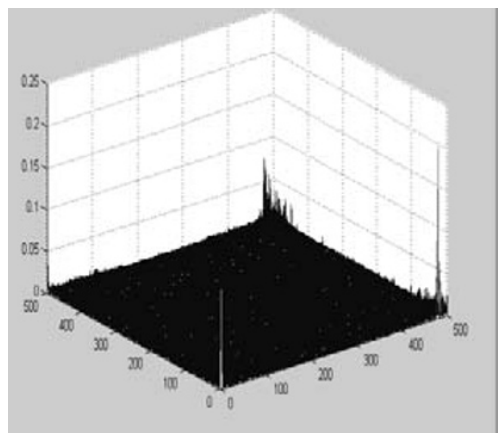

(b) correlation result

Fig.4: Result of rotating angle of 30

From above translation experiment and rotation experiment, in order to make it clearly we sum up the comparison as following table:

Table 1. Comparison results of different related content

\begin{tabular}{cccc}
\hline \multicolumn{2}{c}{ Translation peak values } & \multicolumn{2}{c}{ Rotation peak values } \\
\hline Half related & Quarter related & Rotating $5^{\circ}$ & Rotating $30^{\circ}$ \\
0.791 & 0.493 & 0.3852 & 0.2103 \\
\hline
\end{tabular}

\section{CONCLUSIONS}

In the paper, it takes one-dimensional barcodes for example, and makes use of MATLAB tool to the phase-correlation algorithm simulation. The two experimental results prove the feasibility and effectiveness of the method. The difficult issues such as rotation and defaced which traceability barcode may arise are well solved. So that it will ensure that the food industry can be promptly identified and recall the products which may exist security risks, enhancing the confidence of consumers. Therefore, it will have a very wide application prospect, and make contribution to the traceability of agricultural products

\section{REFERENCES}

G.Wolberg,S.Zokai. Roubust image registration using log-polar transform[A]. Proc. IEEE Int. Conf. Image Processing[C].Vancouver, BC,Canda:IEEE, 2000:493-496.

Harold S S, Michael T Orchard, Ee2Chien Chang, et al. A fast direct Fourier based algorithm for subpixel registration of images [J]. IEEE Transactions on Geoscience and Remote Sensing , 2001,39 (10) : 2235-2243. 
Harold S S, Robert Wolpov. Blind cross-spectral image registration using prefiltering and Fourier-based translation detection [J]. IEEE Transactions on Geosciences and Remote Sensing,2002, 40 (3) : 637-650.

K.Kobayashi,H.Nakajima.T.Aoki.M.Kawamata.T.Higuchi. Principals of phase only correlation and applications. ITE Technical Report, 1996, 20(41):2-4

Ni B, Paulsen MR, Reid J F. Corn kernel crown shape identification using image processing $[\mathrm{J}]$. Trans of the ASAE, 1997, 40(3) :833-838.

Srinivasa R B, Chatterji B N. An FFT-based technique for translation, rotation, and scaleinvariant image registration $[\mathrm{J}]$. IEEE Transactions on Image Processing, 1996, 5 (8) : 1266-1271.

Tillett R D. Image analysis for agricultural processes: A review of potential opportunities[J ] . J Agric Engng Res, 1991, 50: 247-258. 